\title{
Correction to: Ownership influences on corporate social responsibility in the Indian context
}

\author{
James J. Cordeiro ${ }^{1}$ - Ambra Galeazzo ${ }^{2}$. \\ Tara Shankar Shaw ${ }^{3}$ - Rajaram Veliyath ${ }^{4}$. \\ M. K. Nandakumar ${ }^{5}$
}

Published online: 12 April 2018

(C) Springer Science+Business Media, LLC, part of Springer Nature 2018

\section{Correction to: Asia Pac J Manag https://doi.org/10.1007/s10490-017-9546-8}

The original version of this article unfortunately contained a mistake. Beta symbols were missing in the equation under "Analysis and results" heading. Below is the correct equation:

$$
\operatorname{CSR}_{i t}=\beta_{0}+\beta_{1} \text { Ownership } \text { Type }_{i t-1}+\gamma X_{i t-1}+\text { YearFE }+ \text { Industry } F E+\varepsilon_{i t}
$$

M. K. Nandakumar

nmkveettil@gmail.com

James J. Cordeiro

jcordeir@brockport.edu

Ambra Galeazzo

ambra.galeazzo@unipd.it

Tara Shankar Shaw

tara.shankar.shaw@gmail.com

Rajaram Veliyath

rveliyat@kennesaw.edu

1 School of Business, State University of New York, Brockport, 115B Hartwell Hall, 350 New Campus Drive, Brockport, NY 14420, USA

2 Dipartimento Di Scienze Economiche E Aziendali "Marco Fanno", University of Padova, Via Del Santo, 33 Padova, Italy

3 Indian Institute of Technology (IIT) Bombay, Adi Shankaracharya Marg, Powai, Mumbai, Maharashtra 400076, India

4 Coles College of Business, Kennesaw State University, Kennesaw, GA 30144, USA

5 Indian Institute of Management Kozhikode, IIMK Campus P. O., Kozhikode, Kerala PIN - 673 570 , India 